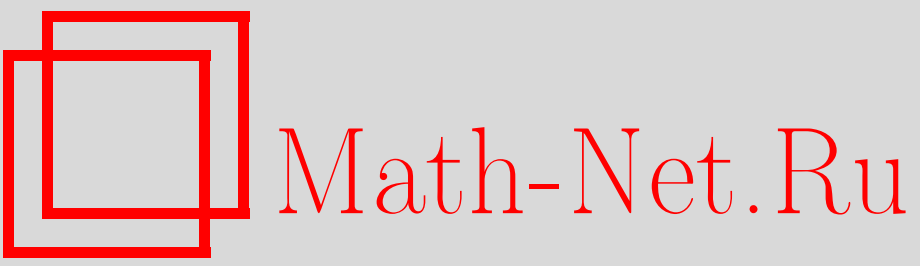

С. М. Натанзон, Формальный ряд для $\tau$-функции, реализующей теорему Римана об областях комплексной плоскости, УМH, 2001, том 56, выпуск 4, 155-156

DOI: https://doi.org/10.4213/rm430

Использование Общероссийского математического портала Math-Net.Ru подразумевает, что вы прочитали и согласны с пользовательским соглашением

http://www.mathnet.ru/rus/agreement

Параметры загрузки:

IP: 54.81 .137 .203

26 апреля 2023 г., 15:15:40 


\section{ФОРМАЛЬНЫЙ РЯД ДЛЯ $\tau$-ФУНКЦИИ, РЕАЛИЗУЮЩЕЙ ТЕОРЕМУ РИМАНА ОБ ОБЛАСТЯХ КОМПЛЕКСНОЙ ПЛОСКОСТИ}

\section{M. НАТАНЗОН}

1. Пусть $D_{+}-$содержащая 0 связная область с аналитической границей на комплексной плоскости $\mathbb{C}$. Тогда согласно теореме Римана существует аналитическая функция $w(z)=\frac{1}{r} z+$ $\sum_{j=0}^{\infty} p_{j} z^{-j}$, переводящая $D_{-}=\mathbb{C} \backslash \bar{D}_{+}$во внешность единичного круга $\{w \in \mathbb{C}|| w \mid>1\}$. Согласно [1] эта функция задается равенством

$$
\log w=\log z-\partial_{t_{0}}\left(\frac{1}{2} \partial_{t_{0}}+\sum_{k \geqslant 1} \frac{z^{-k}}{k} \partial_{t_{k}}\right) v
$$

где $v=v\left(t_{0}, t_{1}, \bar{t}_{1}, t_{2}, \bar{t}_{2}, \ldots\right)$ - функция от бесконечного числа переменных

$$
t_{0}=\frac{1}{\pi} \int_{D_{+}} d^{2} z, \quad t_{k}=\frac{1}{\pi k} \int_{D_{-}} z^{-k} d^{2} z,
$$

удовлетворяющая бездисперсионному пределу иерархии двумерной цепочки Тода:

$$
\begin{aligned}
(z-\xi) e^{D(z) D(\xi) v} & =z e^{-\partial_{t_{0}} D(z) v}-\xi e^{-\partial_{t_{0}} D(\xi) v} \\
(\bar{z}-\bar{\xi}) e^{\bar{D}(\bar{z}) \bar{D}(\bar{\xi}) v} & =\bar{z} e^{-\partial_{t_{0}} \bar{D}(\bar{z}) v}-\bar{\xi} e^{-\partial_{t_{0}} \bar{D}(\bar{\xi}) v} \\
1-e^{-D(z) \bar{D}(\bar{\xi}) v} & =\frac{1}{z \bar{\xi}} e^{\partial_{t_{0}}\left(\partial_{t_{0}}+D(z)+\bar{D}(\bar{\xi})\right) v}
\end{aligned}
$$

и

$$
D(z)=\sum_{k \geqslant 1} \frac{z^{-k}}{k} \partial_{t_{k}}, \quad \bar{D}(\bar{z})=\sum_{k \geqslant 1} \frac{\bar{z}^{-k}}{k} \partial_{\bar{t}_{k}} .
$$

Таким образом, для построения функции $w(z)$ достаточно найти представление функции $v$ в виде ряда Тейлора

$$
v=\sum N\left(i\left|i_{1}, \ldots, i_{k}\right| \bar{i}_{1}, \ldots, \bar{i}_{\bar{k}}\right) t_{0}^{i} t_{i_{1}} \cdots t_{i_{k}} \bar{t}_{\bar{i}_{1}} \cdots \bar{t}_{\bar{i}_{\bar{k}}}
$$

Для $i_{\alpha}, \bar{i}_{\beta} \leqslant 2$ числа $N\left(i\left|i_{1}, \ldots, i_{k}\right| \bar{i}_{1}, \ldots, \bar{i}_{\bar{k}}\right)$ найдены в [1]. В настоящей работе получены рекуррентные соотношения, позволяющие найти все коэффициенты $N\left(i\left|i_{1}, \ldots, i_{k}\right| \bar{i}_{1}, \ldots, \bar{i}_{\bar{k}}\right)$.

2. Положим $\partial_{i}=\partial_{t_{i}}=\frac{\partial}{\partial t_{i}}$ и $\bar{\partial}_{i}=\partial_{\bar{t}_{i}}=\frac{\partial}{\partial \bar{t}_{i}}$.

Пусть $P_{i j}\left(s_{1}-1, \ldots, s_{m}-1\right)$ - число разложений вида $\left\{i=i_{1}+\cdots+i_{m} \mid 1 \leqslant i_{k} \leqslant s_{k}-1\right.$, $k=1, \ldots, m\}$ числа $i$.

Положим $T_{i j}\left(p_{1} \ldots p_{m}\right)=\sum_{n_{1}+\cdots+n_{k}=m} \frac{(-1)^{m+1}}{m} \frac{1}{n_{1} ! \cdots n_{k} !} P_{i j}\left(p_{1}+\cdots+p_{q_{1}}-1, \ldots\right.$, $\left.p_{q_{k-1}+1}+\cdots+p_{q_{k}}-1\right)$, где $q_{j}=\sum_{i=1}^{j} n_{i}$. Определим величины

$$
T_{i_{1} \ldots i_{k}}\left(\begin{array}{c}
s_{1} \ldots s_{m} \\
\ell_{1} \ldots \ell_{m}
\end{array}\right)
$$

Работа выполнена при частичной финансовой поддержке грантов РФФИ-01-01-00739, NWO 047.008.005 и INTAS-00-0259. 
рекуррентными формулами

$$
\begin{aligned}
& T_{i_{1} i_{2}}\left(\begin{array}{cc}
s_{1} \ldots s_{m} \\
1 \ldots & 1
\end{array}\right)=T_{i_{1} i_{2}}\left(s_{1} \ldots s_{m}\right),
\end{aligned}
$$

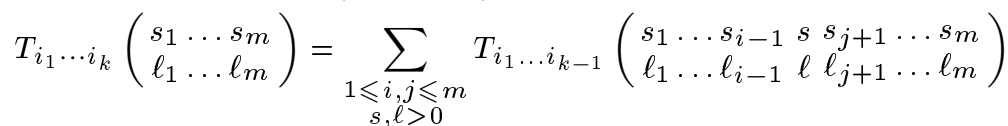

$$
\begin{aligned}
& \times T_{s i_{k}}\left(s_{i}, s_{i+1}, \ldots, s_{j}\right) \frac{\ell !}{\left(\ell_{i}-1\right) ! \cdots\left(\ell_{j}-1\right) !},
\end{aligned}
$$

где $s=s_{i}+s_{i+1}+\cdots+s_{j}-i_{k}, \ell=\left(\ell_{i}-1\right)+\cdots+\left(\ell_{j}-1\right)$.

При $\sum_{j=1}^{k} i_{j} \neq i$ или $\sum_{j=1}^{\bar{k}} \bar{i}_{j} \neq i$ положим $N_{i}\left(i_{1} \ldots i_{k} \mid \bar{i}_{1} \ldots \bar{i}_{\bar{k}}\right)=0$. В противном случае, положим

$$
\begin{aligned}
& N_{i}\left(i_{1} \ldots i_{i_{k}} \mid \bar{i}_{1} \ldots \bar{i}_{\bar{k}}\right)=\sum_{m=1}^{\infty}\left(\sum_{\begin{array}{c}
m \\
s_{1}+\cdots+s_{m}=i_{1}+\cdots+i_{k} \\
\ell_{1}+\cdots+\ell_{m}=m+k-2 \\
s_{j}, \ell_{j} \geqslant 1
\end{array}} i_{1} \cdots i_{k} \bar{i}_{1} \cdots \bar{i}_{\bar{k}}\right. \\
& \left.\times \frac{\left(s_{1}-1\right) ! \cdots\left(s_{m}-1\right) !}{\left(s_{1}-k+1\right) ! \cdots\left(s_{m}-k+1\right) !} \cdot T_{i_{1} \ldots i_{k}}\left(\begin{array}{c}
s_{1} \ldots s_{m} \\
\ell_{1} \ldots \ell_{m}
\end{array}\right) S_{\bar{i}_{1} \ldots \bar{i}_{\bar{k}}}\left(s_{1}, \ldots, s_{m}\right)\right),
\end{aligned}
$$

где $S_{\bar{i}_{1} \ldots \bar{i}_{\bar{k}}}\left(s_{1}, \ldots, s_{m}\right)$ - число разбиений множества $\left\{\bar{i}_{1}, \ldots, \bar{i}_{\bar{k}}\right\}$ на группы $\left\{j_{1}^{p}, \ldots, j_{n_{p}}^{p}\right\}$ $(p=1, \ldots, m)$ такие, что $\sum_{\alpha=1}^{n_{p}} j_{\alpha}^{p}=s_{p}$.

TeOpema.

$$
\begin{aligned}
& v=\sum_{\substack{i_{1}<\cdots<i_{k} \\
\bar{i}_{1}<\cdots<\bar{i}_{\bar{k}}}} \sum_{n_{j}, \bar{n}_{j}=1}^{\infty} \frac{1}{n_{1} ! \cdots n_{k} ! \bar{n}_{1} ! \cdots \bar{n}_{\bar{k}} !} N_{i}\left(\begin{array}{cc|cc}
i_{1} \ldots & i_{k} & \bar{i}_{1} \ldots & \bar{i}_{\bar{k}} \\
n_{1} \ldots & n_{k} & \bar{n}_{1} \ldots & \bar{n}_{\bar{k}}
\end{array}\right) \\
& \times t_{0}^{i-(k+\bar{k})+2} t_{i_{1}}^{n_{1}} \cdots t_{i_{k}}^{n_{k}} \bar{t}_{\bar{i}_{1}}^{\bar{n}_{1}} \cdots \bar{t}_{\bar{i}_{\bar{k}}}^{\bar{n}_{\bar{k}}},
\end{aligned}
$$

əде

$$
N_{i}\left(\begin{array}{cccccc}
i_{1} & \ldots & i_{k} & \bar{i}_{1} & \ldots & \bar{i}_{\bar{k}} \\
n_{1} & \ldots & n_{k} & \bar{n}_{1} & \ldots & \bar{n}_{\bar{k}}
\end{array}\right)=0
$$

если $i \neq \sum_{j=1}^{k} n_{j} i_{j}$ или $i \neq \sum_{j=1}^{\bar{k}} \bar{n}_{j} \bar{i}_{j}$. В противном случае

$$
N_{i}\left(\begin{array}{c|cc}
i_{1} \ldots i_{k} & \bar{i}_{1} \ldots \bar{i}_{\bar{k}} \\
n_{1} \ldots n_{k} & \bar{n}_{1} \ldots \bar{n}_{\bar{k}}
\end{array}\right)=N_{i}\left(i_{1} \ldots i_{1} i_{2} \ldots i_{2} \ldots i_{k} \ldots i_{k} \mid \bar{i}_{1} \ldots \bar{i}_{1} \bar{i}_{2} \ldots \bar{i}_{2} \ldots \bar{i}_{\bar{k}} \ldots \bar{i}_{\bar{k}}\right),
$$

где в последней скобке число $i_{j}$ (соответственно, число $\bar{i}_{j}$ ) встречается $n_{j}$ (соответственно $\bar{n}_{j}$ ) раз.

\section{СПИСОК ЛИТЕРАТУРЫ}

[1] I. K. Kostov, I. Krichever, M. Mineev-Weinstein, P. B. Wiegmann, A. Zabrodin. $\tau$-function for analytic curves // arXiv:hep-th/0005259.

Московский государственньй университет им. М. В. Ломоносова, Независимый московский университет

Принято редколлегией E-mail: natanzon@mccme.ru 